\title{
UAV Trials for Multi-Spectral Imaging Target Detection and Recognition in Maritime Environment
}

\author{
Hugo Silva, José Miguel Almeida, Flávio Lopes, J.P. Ribeiro, Sara Freitas, \\ Guilherme Amaral, Carlos Almeida, Alfredo Martins, Eduardo Silva \\ INESC TEC Institute for Systems and Computer Engineering of Porto \\ ISEP - School of Engineering, Porto Polytechnic Institute, Porto, Portugal \\ Email: hugo.m.silva, jose.m.almeida, fmlopes,jmpr,scf, gasilva,aom,carlos.almeida,eduardo.silva @inesctec.pt
}

\begin{abstract}
This paper addresses the use of heterogeneous sensors for target detection and recognition in maritime environment. An Unmanned Aerial Vehicle payload was assembled using hyperspectral, infrared, electro-optical, AIS and INS information to collect synchronized sensor data with vessel ground-truth position for conducting air and sea trials. The data collected is used to develop automated robust methods for detect and recognize vessels based on their exogenous physical characteristics and their behaviour across time. Data Processing preliminary results are also presented.
\end{abstract}

Keywords-Target Detection, Target Tracking, Hyperspectral Imaging, Infrared Imaging, Unmanned Aerial Vehicle, remote sensing, georeferencing

\section{INTRODUCTION}

The need of using autonomous systems in this case Unmanned Aerial Vehicles (UAVs) for maritime border surveillance is becoming more relevant due to the lack of piloted means and the need to achieve 24/24 hours surveillance. In civil applications, its important that the UAVs cope with the capability to automatically detect and recognize attributes of the type of vessel and also classify their behaviour. The goal is to use the UAV to perform active sensing by processing heterogeneous sensor information on the fly in order to be able to detect and track targets in flight.

One of simplest but at the same time technically complex way of achieving such objective, is to carry a sensor payload that enables the designed system to cope with the extraction of physical characteristics of the target in order to extract vessel information, e.g. shape, type, dimensions, colour, cargo or even type of vessel building material. To do so, all of this information needs to be registered and synchronized within a common framework to be useful not only for on-board data processing but also for trasmission to other centres of data processing on the ground.

The use of vision sensors in the visible [1] [2] and infrared spectre [3] [4] [5], is becoming widespread for surveillance operations. However, most of the UAVs that carry this type of sensor payload use for streaming video to land for human classification of the target, while others record data that is only processed after landing. There are few applications which goal is to perform an automated real-time detection and recognition of vessels in maritime environment. With the objective to pre-process all data on-board and then disseminate it to other higher hierarchical abstraction levels. Specially, if one considers the heterogeneous use of visual sensors cameras with different image spectres e.g. visible, infrared and hyperspectral imaging at the same time.

In this work, we present a UAV multi-spectral image acquisition setup that is used in the detection and classification of maritime targets in real-time. The objective was to equip a UAV with reasonable payload capability $\approx 15 \mathrm{~kg}$, with a sensor payload capable of collecting visible, infrared and hyperspectral imaging information combined with a common synchronized GPS based timestamp. The payload also contains a Inertial Navigation System that provides the UAV position, velocity and attitude to the recording system, as well as, an Automatic Identification System (AIS) receiver to record the emitter vessel periodical AIS messages.

To validate our data acquisition setup, we conduct air and sea trials in collaboration with the Portuguese Air Force and the Portuguese Navy to record datasets for algorithm development. For purposes of ground-truth and to be able to correctly geo-reference the vessel coordinates, a RTK GPS receiver, was placed in the target vessel to instantly recorded the vessel position during the UAV missions. Preliminary results of the target detections using the data collect by all sensors in the air and sea trials are displayed in the results section.

The remaining of the paper has the following organization: Section II contains the sensor payload description, in section III a brief introduction to type of data processing that is required for each individual sensor is presented. In section IV we describe the sea and air trials data collection and some results obtained from individually processing each sensor data. Finally, in section $\mathrm{V}$ we present some conclusions of the results obtained and detail some of the future steps of our research.

\section{PAYLOAD DESCRIPTION}

In this section, we are going to describe the payload hardware and its main characteristics. The sensor and data collection hardware contains the following components:

- A CPU unit (Intel NUC ML100G-30 i5 $2.30 \mathrm{GHz}$ ): that is used to record the E/O camera images, IR images and AIS transmitted messages;

- $\quad$ A E/O camera (Point-Grey BFLY-PGE-31S4C-C 3.2 MP): that acquires 24-bit RGB images, with synchronized timestamp. 
- IR camera (Xenics Gobi-640-CL): that acquires images in the infrared spectrum, 640x480 resolution, with synchronized timestamp;

- $\quad$ Frame Grabber (Pleora CL-U3): Acquires frames from the infrared camera via Camera Link interface, making them accessible to the processing unit by USB3.0.

- $\quad$ AIS (Yacht AIS100PRO): receive data from the transmitter AIS installed on vessels.

- $\quad$ Processing unit (SPECIM DPU): for data acquisition of the hyperspectral camera, inertial navigation and GPS information;

- Hyperspectral camera (SPECIM AisaKESTREL 16): acquires data in the electromagnetic spectrum from ultraviolet to long-infrared.

- Inertial and GPS (OXTS xNav550): dual GNSS receivers and a Inertial measurement unit.

- Wi-fi communications: $700 \mathrm{MHz}$ module, for ground UAV payload communications. Used for start/stop recording and debug purposes.

- External antennas (antenna Wi-Fi, antenna GPS and antenna AIS).

- Power supply: LifePO4 battery and DC/DC for regulated voltage.

In Figure 1 is possible to see the payload wiring diagram where all sensors and equipments wiring connections are illustrated. Our system consists of two processing units. The NUC $\mathrm{PC}$ runs a Ubuntu 14.04 operating system environment with the Robotic Operating System(ROS) as middleware framework. This equipment is used to acquire and process images from the E/O camera, IR Camera, and also from the AIS receiver. It also records Inertial Measurements from the IMU unit via Ethernet connection for geo-reference all the data to the UAV position. In parallel to this setup, we have the hyperspectral camera acquisition setup that contains, the other DPU that currently runs a Windows 7 operation system and is used to receive and trigger the image acquisition of the hyperspectral camera and also receive the IMU sensor information via serial port. The two processing units are synchronized using the signal PPS received from the OXTS system.

In Figure 2, we can see the payload as it is assembled and mounted inside the UAV. The sensors are all placed in the bottom structure so that all the cameras can be placed near/outside the bottom fuselage of the UAV. While the data recording CPUs, for the EO camera and the IR camera and the hyperspectral are placed on top layer.

\section{SENSORS}

In this section we will introduce what type of processing is going to be performed with each sensor information, and how this information will be combined into a integrated data acquisition/processing setup.

\section{A. Hyperspectral Camera}

The use of an hyperspectral camera is becoming ubiquitous in remote sensing operations [6], [7]. Its use in data collection

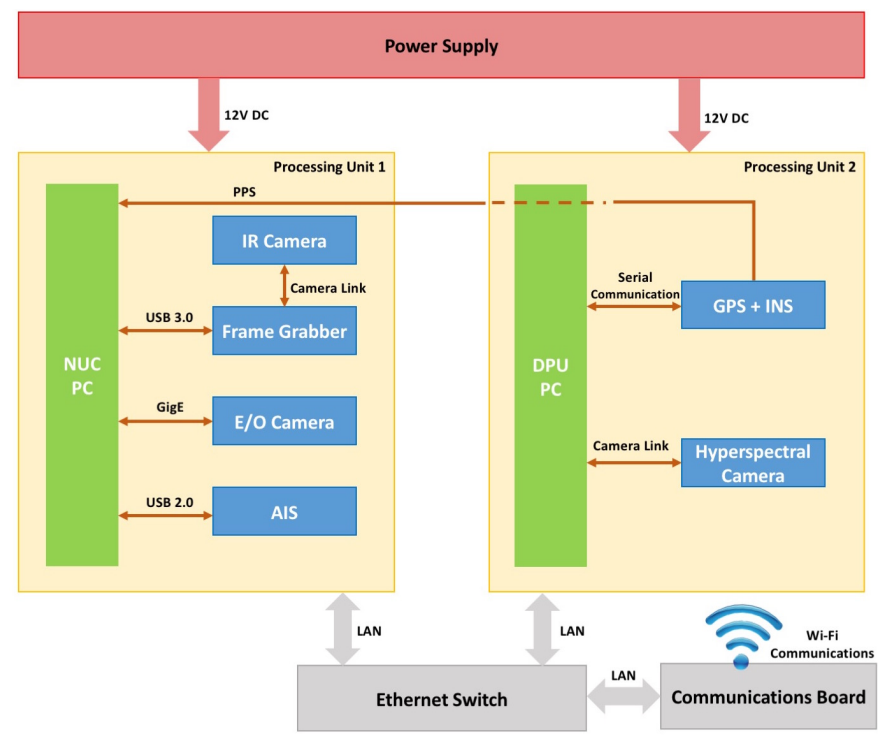

Fig. 1. Payload Wiring diagram

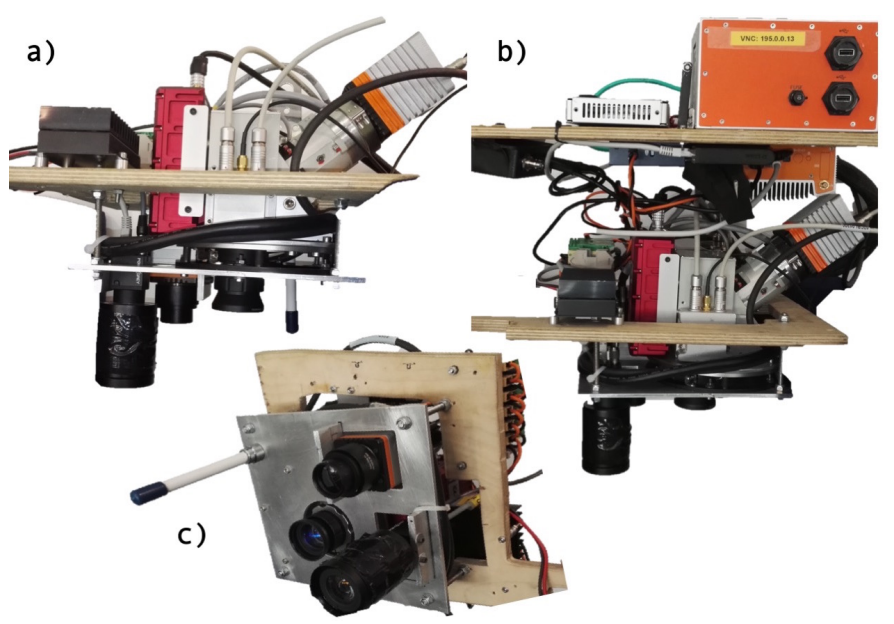

Fig. 2. UAV payload used in the data collection trials: a) Side view of the payload, with imaging sensors and IMU unit; b) Side view of the full payload; $c$ ) Bottom view of the payload, showing all imaging sensors and the AIS antenna.

campaigns is becoming frequent in applications that range from agriculture, landscaping geo-referencing to others such as surveillance and inspection tasks [8],[9]. In our maritime target detection, recognition and identification application, hyperspectral data allows us to acquire image data of a target scene over the electromagnetic spectrum, from ultraviolet to long-infrared. Based on this information, we are able to obtain detailed information about the scene, which facilitates the detection of the vessel building material e.g fibre, aluminium, wood, rubber, persons on-board and also the cargo carried by the vessel. When all image data is associated, it generates a hyperspectral image cube, consisting of a set of images layered on top of one another, as can be seen in Figure 3. Each image corresponds to one wavelength. Therefore, the hyperspectral cube has a spectral dimension equal to the number of data wavelengths that the hyperspectral camera is able to acquire.

Given that an image corresponds to one wavelength (in 


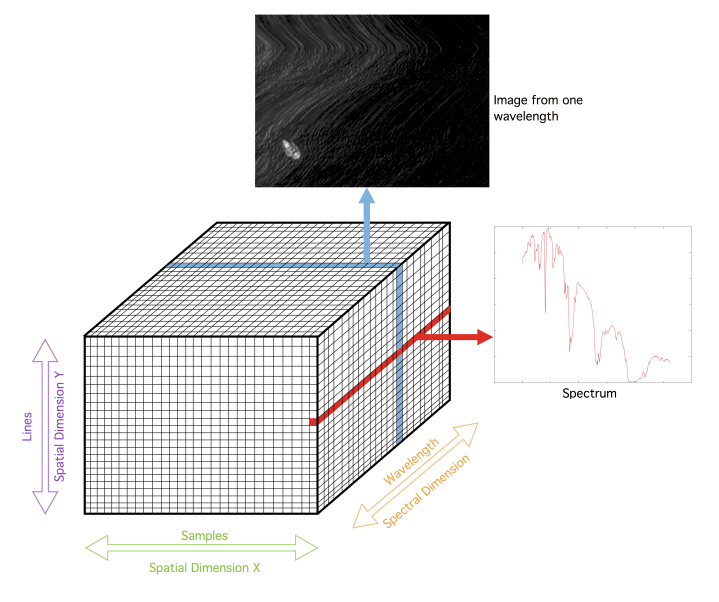

Fig. 3. Hyperspectral Cube

spectral dimension $\lambda$ ), each pixel (in spatial dimension $\mathrm{XY}$ ) consists of a spectrum over the different wavelengths observed by the camera. This characteristic is named "spectral signature". If one compares the spectral signature to a previously known material one can identify the type of vessel building material.

There are two distinct ways of detecting a target using a hyperspectral data: using a pushbroom camera or by using a tunable spectral filter. In this work, we used a hyperspectral pushbroom camera.

The pushbroom camera allows to collect simultaneously full spectral data using a single scan line over time. The sensor is able to acquire all spectral information exactly at the same time, while being insensitive to sensor/sample movement. Also, there is no moving parts in the sensor, and so it can collect light from sample to camera 5 to 20 times more efficiently than a tunable spectral filter. Furthermore, only a line across the sample needs to be illuminated, which leads to 10 to 30 times more light and consequently more speed. So, the data is received line by line, for all wavelengths at the same time. Which means that for a single scan line, we are to able to obtain the spectrum response for each pixel.

However, when you get data from the hyperspectral sensor camera, these spectral signature is nothing more than raw data, i.e., digital numbers. This measures are commonly used to describe pixel values that have not yet been calibrated into physically meaningful units. This means that if we want a material spectrum, this values need to be corrected in order to obtain a measurement of radiance or reflectance. In hyperspectral imaging, when we have raw data, this means that this values have the influence of different factors, such as: light source, sensor, atmosphere and surface material. The only factor that contains only characteristics of the material is the surface material, whereas all others should be eliminated.

Radiance refers to the amount of radiation coming from an area. In order to derive a radiance value from a digital number, a gain and offset must be applied. In hyperspectral imaging, this calculation is performed for all pixels, and the gain and offset to be applied depend on the wavelength. This values are typically retrieved from the images metadata or received from the data provider. Radiance values normally have units of $\mathrm{w} /(\mathrm{m} 2 \mathrm{SR} \mathrm{nm})$. Transforming the digital numbers to radiance is called calibration. In order to perform this step, is necessary to calculate the radiance values for each pixel:

$$
L_{\lambda}=\text { Gain } \times \text { Pixelvalue }+ \text { Offset }
$$

With radiance, in addition to surface material, we have influence of light source and atmosphere. In some applications, this value may be sufficient for the desired implementation. However, if we want to get sufficient information about a material spectrum in order to identify which material are we seeing, its necessary to determine the reflectance of the spectrum. Reflectance is the proportion of the radiation striking a surface to the radiation reflected of it.

Our objective for the hyperspectral data use in our maritime target detection application is twofold: First, we want to be able to detect the changes in spectrum pixel by pixel in near real time, that way we can easily try and remove the foreground for the background, meaning vessels from water. This process, can only be achieved since or designated targets are much smaller than the dominant background, thus allowing to detect changes in the radiance spectrum; Second, since the UAV due to payload computational limitations does not have the required computational capability to further process all the hyperspectral data information. We want to store this spectral information of a given target and only transmit the target information to a control centre on the ground where the data can be further processed (in reflectance spectrum), and atmospheric effects will be removed.

For this data to be useful the detected candidate targets (spectrum shifts other than water) need to be georeferenced in terms of their GPS coordinates position at sea. Therefore, we need to be able to relate the pushbroom camera image coordinates, with the obtained target GPS coordinates.

For a pushbroom camera, this can relation can be obtained using the following equation:

$$
\begin{array}{r}
\left(\begin{array}{l}
x \\
y \\
z
\end{array}\right)_{\text {Object }}^{U T M}=f_{\text {geo }}^{U T M}\left(\begin{array}{c}
x \\
y \\
z
\end{array}\right)_{\text {Sensor }}^{g e o}+s \cdot R_{N E D}^{U T M} \cdot \\
\cdot\left(R_{I M U}^{N E D} \cdot R_{\text {Sensor }}^{I M U} \cdot\left(\begin{array}{c}
0 \\
y_{i} \\
-f
\end{array}\right)_{\text {Object }}^{\text {Sensor }}\right)
\end{array}
$$

where:

- $\left(\begin{array}{c}0 \\ y_{i} \\ -f\end{array}\right)_{\text {Object }}^{\text {Sensor }}$ is the vector that corresponds to the target position in the image. $x$ is 0 due to line concept of pushbroom cameras, and $y_{i}$ corresponds to $y_{i}=-\frac{N-1}{2}+i$, where $N$ is the number of samples (pixels) $i$ is the pixel to be analysed. $f$ is the focal length in pixels;

- $\quad R_{\text {Sensor }}^{I M U}$ is the rotation matrix between the IMU and the sensor; This rotation matrix needs to be calibrated or obtained priorly, based on the IMU and sensor 
assembly in terms of IMU position related to the sensor reference frame

- $\quad R_{I M U}^{N E D}$ is the rotation matrix that corresponds to the UAV attitude position in terms of roll, pitch and yaw angles, this information is obtained using the Inertial Measurement Unit;

- $R_{N E D}^{U T M}$ is the rotation matrix from the IMU (NED reference frame) which is the UAV reference frame to UTM coordinates that are used as world target coordinates.

- $\quad s$ corresponds to the scale factor. The scale factor by approximation using the Ground sampling distance, which corresponds to the resolution that is on the floor. The Ground sampling distance in $y$ is given by: $G S P_{y}=\Delta y \cdot m_{b}=\Delta y \cdot \frac{h}{f}$ where $\Delta y$ is the pixel size and $\frac{h}{f}$ is considered an approximation for the scale ( $h$ is the altitude of the UAV, and $f$ is the focal length in pixels). Other way of obtaining this value is if we use stereo vision techniques or a Digital Evelevation Model (DEM);

- $f_{\text {geo }}^{U T M}\left(\begin{array}{l}x \\ y \\ z\end{array}\right)_{\text {Sensor }}^{\text {geo }}$ is the vector that contains the UAV position at the time of the detection in World Coordinates (UTM);

Based on this equation and in the spectral analysis procedure in terms of radiance, we are able to separate the foreground from the background pixels and establish a relation between the hyperspectral camera position and the matching spectral information of a candidate target at sea in real time.

\section{B. Infrared Camera}

The infrared camera is probably the most utilized sensor for vessel detection using UAV at sea. Its high contrast foreground/background pixels makes target vessels easily detectable for intensity, saliency based image processing methods allowing easy target detection with a low false positive rate. The infrared camera utilized is a LWIR camera, and it was calibrated based on a procedure detail in [10].

\section{Electro Optical Camera}

For the Electro Optical Camera, we utilized a low sensitivity Pointgrey Blackfly camera. The E/O camera was a dual purpose use in our application: First, is to extract static images of a potential target to transmit it to land for human classification; Second, use the collected images for process and identify possible target candidates in the image (using saliency based and feature based methods) that can be feed to a higher level hierarchical classifier that discriminates if its a target or not based on its extrinsic characteristics and trajectory behaviour.

\section{Navigation}

For Navigation purposes, the system used the hyperspectral system Inertial Measurement Unit, that was configured to provide the scan line acquisition inertial measurements (NCOM messages) via serial port to the DPU, and also to provide the

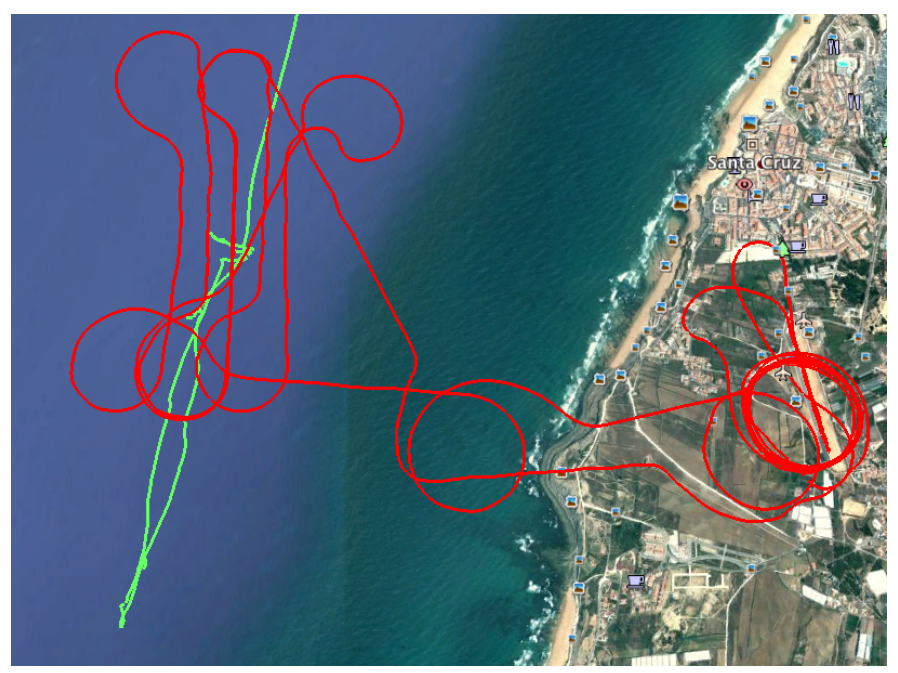

Fig. 4. UAV trajectory (red line) and vessel trajectory (green line)

other CPU with the inertial measurements (NCOM messages) via Ethernet port.

For synchronizing both the processing units the PPS signal generated by GPS/IMU system is received in both units. We used the gpsd service daemon [11] for monitoring and receive the gps data. With the data received in the gpsd and the pps signal, we then use the Chrony clock synchronization daemon [12] in order to synchronize internal clock of the processing unit to GPS time, assuring precise timing information. In relation to the synchronization between the IR camera and the EO camera, an external trigger out generated by the IR camera acts like an input to trigger the E/O camera, thus obtaining synchronized images between the two E/O and IR cameras.

\section{AIR AND SEA TRIALS}

The air and sea trials took place in Santa Cruz, Peniche in the west coast of Portugal over a week, and consisted on a UAV performing a pre-defined flight plan carrying a heterogeneous sensor payload and record synchronized information of an hyperspectral, infrared and electro-optical camera, as well as, AIS and Inertial Navigation System Information.

\section{A. Missions}

The missions were performed to collect synchronized data of three types of cameras as well as inertial navigation information. The missions consisted on having a UAV performing U-shape movements at approximately 300 meters high above sea level, while passing over the vessel that was acting as as intended target, as seen in Figure 4.

In Figure 4, we can see in red the UAV trajectory, and in green the vessel trajectory. The vessel was equipped with is own GPS-RTK receiver collecting GPS data for external ground-truth validation.

In terms of flight plan a compromise had to be established in order to perform the data collection. The performed trajectory is far from ideal for detection purposes with the $\mathrm{E} / \mathrm{O}$ and infrared Camera since the UAV goes over the vessel in a few seconds and so the E/O and IR cameras have few 

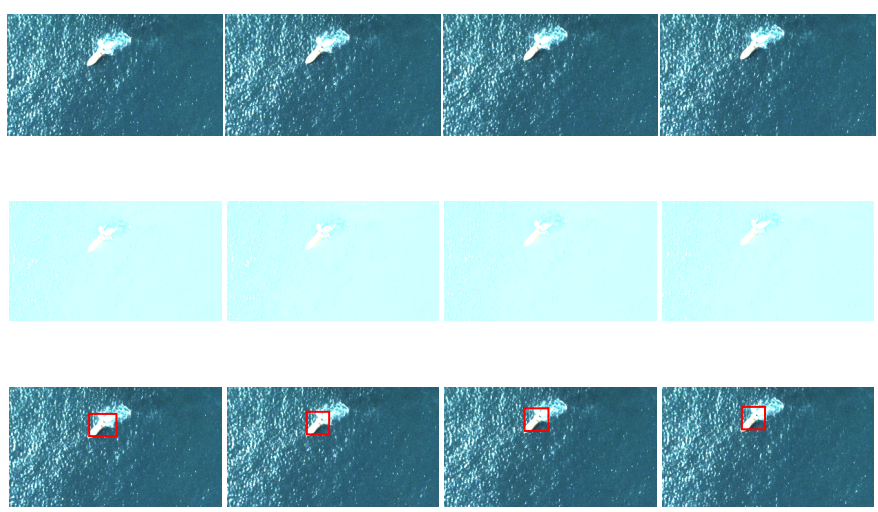

Fig. 5. Results for E/O camera: each image corresponds to the detection of vessels based on a combination of color, intensity and entropy detectors. In the middle row, we can see the image complement of the output of the detection.

image frames with vessels in the image for each passage. However, our intentioned objective was to put the UAV in a direct route over the vessel, so hyperspectral data could be directly georeferenced and compared with visible spectre data. In order to foster algorithm development and spectral evaluation of materials using hyperspectral data.

\section{B. Results}

The data collection allows us to individually start to develop image processing methods for the three types of images that were collected. For the E/O images, we utilize saliency detectors based on colour, intensity and entropy at different images scales, to separate the foreground pixels belonging to the vessel from the background. Even though we worked for most of the image sequences, see Figure 5. The detection in the E/O needs to be more robust to wave crest effects and sun glare on the water, that produce image saturation, on the vessel current trajectory. Temporal analysis consistency and motion compensation algorithms are currently being implemented to diminish the effects of these image artefacts in the output of the $\mathrm{E} / \mathrm{O}$ iamge processing pipeline. These detections even though quite simple to conduct in the image processing domain are to be performed in real-time on-board the UAV, and their outputs used to feed a ground station classifier that will perform the recognition of the vessel type based on these detections and target image crops.

In the infrared image domain the objective is to detect the vessel(s) in the image sequence and obtain the vessel size. The vessel detection in infrared images (LWIR camera) is intensity based, and an adaptive threshold is used to detect the vessel in the images and discard other artefacts based on their size and consistency over time. The precision rate of detection is near $100 \%$, we only drop the detections that go across the image boundaries, see Figure 6 . In future work these detections will also be checked for temporal consistency.

As for the hyperspectral detection, the develop image processing methods consists on analysing a determined band and detect the transitions over the radiance spectrum. We are looking to classify pixels material in a binary manner as being non-water/water pixels. Afterwards, the pixels are geo-referenced, and the raw data of the detections is to be

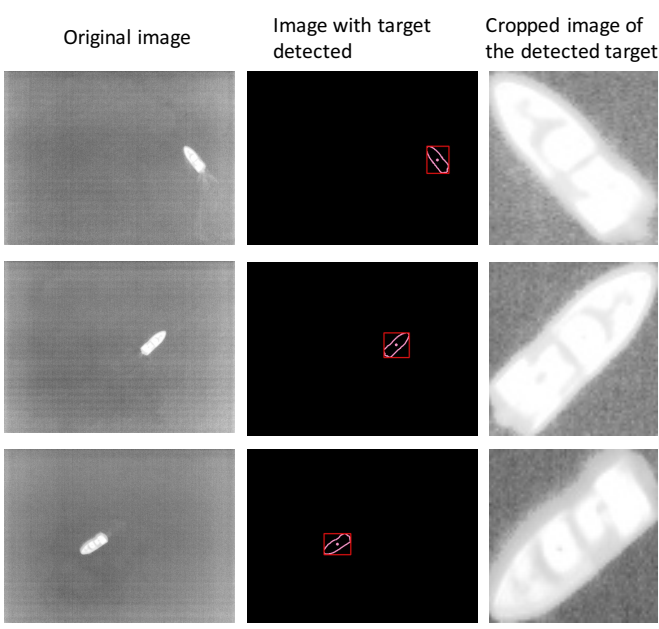

Fig. 6. Results obtained during the target detection phases of the IR cameram, the images refer to three different passages over the vessel.

transmitted to the ground station, where the data can be removed of atmospheric effects and processed in terms of reflectance for material identification, see Figure 7.

The geo-reference results for the hyperspectral limits are still with an average error of $\approx 6$ meters. But this results were obtained without boresight calibration. We are now developing a re-projection method using ground control points to diminish this error.

\section{CONClusions AND Future WORK}

In this work, we presented an heterogeneous sensor setup for target detection and recognition in maritime environment. For being able to perform such task an hyperspectral camera, infrared camera and E/0 camera were assembled in a single UAV payload to perform the target detection. An air and sea campaign was performed to collect the data from all sensors for algorithm development, external ground-truth validation was also obtained during the dataset campaign. The preliminary results indicate that we are able to extract valuable synchronize information of all the sensors, process the sensor data and obtain the target information.

In future work, we will continue to develop image processing methods for processing the images in real-time. We will add temporal consistency and target tracking capabilities to our system. We also plan to transmit the detections and the images crops to a ground station and perform target classification, recognition based on a learning model interaction between the UAV and the ground station.

\section{ACKNOWLEDGMENTS}

The authors would like to thank the Portuguese Air Force and the Portuguese Navy for providing the UAV and the boats for the data collection. The authors would also like to thank SPECIM and XENICS for providing the Hyperspectral camera and the IR camera for the payload used in this dataset campaign.

This work is financed by the ERDF - European Regional Development Fund through the Operational Programme for 

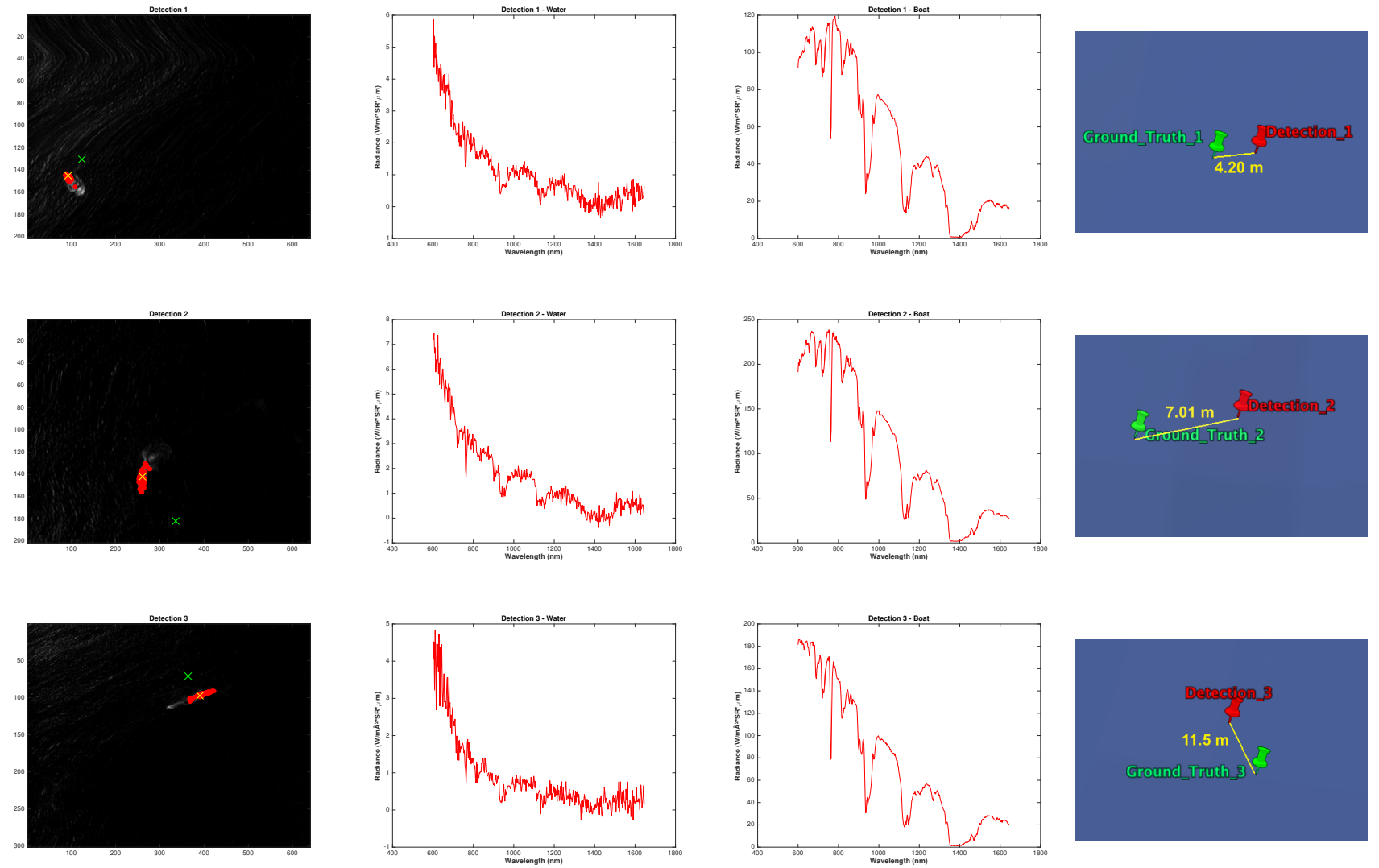

Fig. 7. Results for hyperspectral camera: each line of pictures corresponds to one detection. The first column displays the image obtained using the band 20 of the data obtained. The cross yellow represents the point analysed to obtain the spectrum of the boat, while the cross green mark the point at what was obtained the spectrum of water. The points in red represent the anomalies detected in relation to the background. The second column shows the water spectrum for the three detections. This spectrum is related to the green cross described above. The third column shows the boat spectrum for the three detections. This spectrum is related to the yellow cross initially described. The last column shows the projection of the points in the world. The green is the point of ground truth and the red is the projected point obtained. In yellow is the distance between the two points, in meters.

Competitiveness and Internationalisation - COMPETE 2020 Programme within project POCI-01-0145-FEDER-006961, by the SUNNY Project funded by the European Commission under the FP7 programme Grant Agreement number: 313243, and by National Funds through the FCT - Fundacao para a Ciencia e a Tecnologia (Portuguese Foundation for Science and Technology) as part of project UID/EEA/50014/2013.

\section{REFERENCES}

[1] W. Kruger, Z. Orlov, "Robust Layer-based Boat Detection and Multitarget-tracking in Maritime Environments", Proceedings of the 2010 NURC WaterSide Security Conference (WSS), November 3-5, Carrara, Italy, 2010.

[2] S. Fefilatyev, D.B. Goldgof, L. Langebrake, "Towards Detection of Marine Vehicles on Horizon from Buoy Camera", Proc. SPIE, Vol. 6736, Unmanned/Unattended Sensors and Sensor Networks IV, Florence, Italy, 2007.

[3] A. Ondini, G. Marsiglia, L. Fortunato, G. Balzarotti, "Techniques for detection of multiple, extended, and low contrast targets in infrared maritime scenarios", Optical Engineering, Vol. 45, December 2006.

[4] H. Li, X. Wang, "Automatic Recognition of Ship Types from Infrared Images Using Support Vector Machines", International Conference on Computer Science and Software Engineering (CSSE), Vol. 6, pp. 483486, 2008.

[5] G. Cruz, A. Bernardino, "Image Saliency Applied to Infrared Images for Unmanned Maritime Monitoring Computer Vision Systems", 10th
International Conference, ICVS 2015, Copenhagen, Denmark, July 6-9, 2015.

[6] E. Ben-Dor, B. Kindel, A.F.H Goetzb, "Quality assessment of several methods to recover surface reflectance using synthetic imaging spectroscopy data", Remote Sensing of Environment, Volume 90, Pages 389 - 404, ISSN 0034-4257,(2004).

[7] R. Muller, M. Lehner, Rainer Muller, P. Reinartz, M. Schroeder, B. Vollmer, "A program for direct georeferencing of airborne and spaceborne line scanner images", ISPRS Commision I WGI/5, Pages 1 - 3, (2002)

[8] Doneus, M.; Verhoeven, G.; Atzberger, C.; Wess, M. Rus, M. "New ways to extract archaeological information from hyperspectral pixels" Journal of Archaeological Science , 2014, 52, 84 - 96

[9] Lagueux, P.; Puckrin, E.; Turcotte, C. S.; Gagnon, M.A.; Bastedo, J.; Farley, V. Chamberland, M. "Airborne infrared hyperspectral imager for intelligence, surveillance and reconnaissance applications" Proc. SPIE, 2012, 8542, 854226-854226-10

[10] A. Dias, C. Bras, A. Martins, J. Almeida and E. Silva, "Thermographic and visible spectrum camera calibration for marine robotic target detection," 2013 OCEANS - San Diego, San Diego, CA, 2013, pp. $1-5$.

[11] http://www.catb.org/gpsd/

[12] https://chrony.tuxfamily.org 